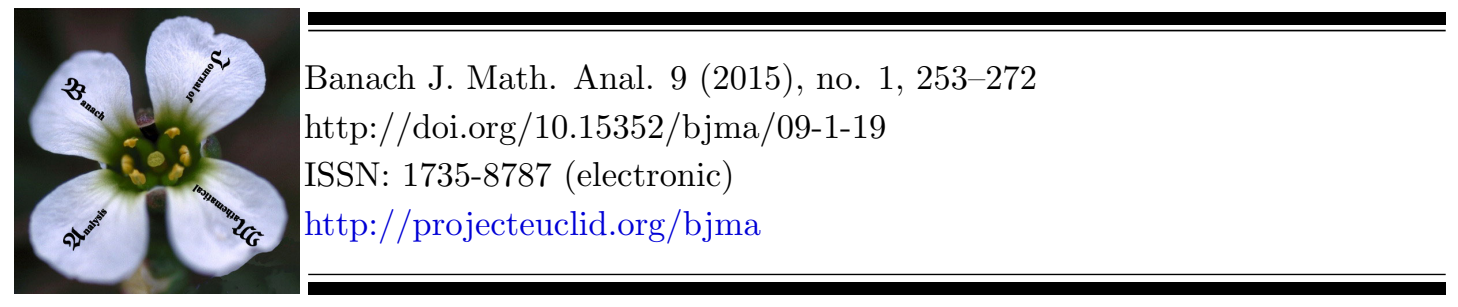

\title{
SKEW SYMMETRIC WEIGHTED SHIFTS
}

\author{
SEN ZHU
}

Communicated by I. B. Jung

\begin{abstract}
An operator $T$ on a complex Hilbert space $\mathcal{H}$ is called skew symmetric if $T$ can be represented as a skew symmetric matrix relative to some orthonormal basis for $\mathcal{H}$. We first give a canonical decomposition for general skew symmetric operators. Based on this decomposition, we provide a classification of skew symmetric weighted shifts.
\end{abstract}

\section{INTRODUCTION AND PRELIMINARIES}

Throughout this paper, we let $\mathbb{C}, \mathbb{Z}$ and $\mathbb{N}$ denote the set of complex numbers, the set of integers and the set of positive integers respectively. $\mathcal{H}$ will always denote a complex separable Hilbert space. We let $\mathcal{B}(\mathcal{H})$ denote the algebra of all bounded linear operators on $\mathcal{H}$.

Definition 1.1. A map $C$ on $\mathcal{H}$ is called an antiunitary operator if $C$ is conjugatelinear, invertible and $\langle C x, C y\rangle=\langle y, x\rangle$ for all $x, y \in \mathcal{H}$. If, in addition, $C^{-1}=C$, then $C$ is called a conjugation. An operator $T$ in $\mathcal{B}(\mathcal{H})$ is called a skew symmetric operator (SSO, for short) if $C T C=-T^{*}$ for some conjugation $C$ on $\mathcal{H}$.

We remark that the terminology stems from the fact that $T$ is skew symmetric if and only if $T$ can be represented as a skew symmetric matrix (that is, $A=-A^{t}$ ) with respect to some orthonormal basis [10, Lem. 1].

The motivation for the study of SSOs stems from the emerging theory of complex symmetric operators on Hilbert space (see [9, 10, 11, 12, 13] for references).

Date: Mar. 26, 2014; Accepted: May 5, 2014.

2010 Mathematics Subject Classification. Primary 47B37; Secondary 47B99, 47A05.

Key words and phrases. Skew symmetric operators, complex symmetric operators, weighted shifts, truncated weighted shifts. 
Definition 1.2. An operator $T$ in $\mathcal{B}(\mathcal{H})$ is called a complex symmetric operator (CSO, for short) if there is a conjugation $C$ on $\mathcal{H}$ so that $C T C=T^{*}$.

Also one can check that $T$ is complex symmetric if and only if $T$ has a symmetric matrix representation with respect to some orthonormal basis. The earliest study of CSOs dates back to the middle of the 20th century [15, 16]. Later, many papers were devoted to the study of CSOs (see [27] and references therein). At the present time, Garcia and Putinar $[10,11]$ has proved many interesting results. The study of CSOs has many motivations in function theory, matrix analysis and other areas; in particular, CSOs are closely related to the study of truncated Toeplitz operators, which was initiated in Sarason's seminal paper [22] and has led to rapid progress in related areas [3, 13, 14, 23, 24].

The following lemma contains some elementary facts about SSOs.

Lemma 1.3 ([20], Lem. 1.4). Let $C$ be a conjugation on $\mathcal{H}$. Denote $S_{C}(\mathcal{H})=$ $\left\{X \in \mathcal{B}(\mathcal{H}): C X C=-X^{*}\right\}$. Then

(i) if $A, B \in \mathcal{B}(\mathcal{H}), C A C=A^{*}$ and $C B C=B^{*}$, then $[A, B]:=A B-B A \in$ $S_{C}(\mathcal{H})$

(ii) if $T \in S_{C}(\mathcal{H})$, then $C T^{2 n} C=\left(T^{2 n}\right)^{*}$ for all $n \in \mathbb{N}$;

(iii) the class $S_{C}(\mathcal{H})$ is norm-closed and forms a Lie algebra under the commutator bracket $[\cdot, \cdot]$;

(iv) if $T \in S_{C}(\mathcal{H})$, then $\sigma(T)=-\sigma(T)$.

By Lemma 1.3 (i), one can use CSOs to construct SSOs. In particular, if $T \in \mathcal{B}(\mathcal{H})$ is complex symmetric, then $T^{*} T-T T^{*}$ is always skew symmetric. By [10, Prop. 3], all truncated Toeplitz operators are complex symmetric with respect to the same conjugation. Then it follows from Lemma 1.3 (i) that any commutator of two truncated Toeplitz operators is skew symmetric. These are important examples of skew symmetric operators.

As already mentioned in the foregoing part, the primary motivation for the study of SSOs lies in its connections to CSOs. From Lemma 1.3 (i) and (ii), one may see this point. It is often difficult to determine whether a given operator is complex symmetric. By Lemma 1.3 (i), if $T$ is complex symmetric, then $T^{*} T-$ $T T^{*}$ is skew symmetric. In view of the description of skew symmetric normal operators [20, Thm. 1.10], this provides another approach to CSOs. In a recent paper [18], one can see such an application to Toeplitz operators. On the other hand, each operator $T$ on $\mathcal{H}$ can be written as the sum of a CSO and an SSO. In fact, arbitrarily choose a conjugation $C$ on $\mathcal{H}$ and set $A=\frac{1}{2}\left(T+C T^{*} C\right)$, $B=\frac{1}{2}\left(T-C T^{*} C\right)$. Then $C A C=A^{*}, C B C=-B^{*}$ and $T=A+B$. This reflects some universality of CSOs and SSOs.

Another motivation for the study of SSOs lies in the connections between SSOs and anti-automorphisms of singly generated $C^{*}$-algebras. Recall that an antiautomorphism of a $C^{*}$-algebra $\mathcal{A}$ is a vector space isomorphism $\varphi: \mathcal{A} \rightarrow \mathcal{A}$ with $\varphi\left(a^{*}\right)=\varphi(a)^{*}$ and $\varphi(a b)=\varphi(b) \varphi(a)$ for $a, b \in \mathcal{A}$. An anti-automorphism 
or an automorphism $\rho$ is said to be involutory if $\rho^{-1}=\rho$. Involutory antiautomorphisms play an important role in the study of the real structure of $C^{*}$ algebras $[2,25,26]$. It is not necessary that each $C^{*}$-algebra possesses an involutory anti-automorphism on it $[4,5,21]$. In a recent paper [30], certain connections between SSOs and anti-automorphisms of singly generated $C^{*}$-algebras are established. In particular, it is proved that if $T$ is skew symmetric, then $C^{*}(T)$ (the $C^{*}$-algebra generated by $T$ and the identity operator $I$ ) admits an involutory anti-automorphism on it (see [30, Cor. 3.2]). Moreover, this is used to classify certain SSOs up to approximate unitary equivalence.

Recently, there has been growing interest in SSOs (see [27, 28, 20, 19, 30]). In [27], Zagorodnyuk studied the polar decomposition of SSOs and obtained some basic properties of SSOs. In [28], Zagorodnyuk studied the skew symmetry of cyclic operators. In [20], Li and the author classified skew symmetric normal operators. In [19], Li and Zhou described skew symmetric partial isometries. In a recent paper [30], the author obtained a classification of certain SSOs up to approximate unitary equivalence.

The main aim of this paper is to classify skew symmetric weighted shifts. Recall that a (forward) weighted shift $T$ on $\mathcal{H}\left(\operatorname{dim} \mathcal{H}=\aleph_{0}\right)$ with weighted sequence $\left\{w_{n}\right\}$ is the operator defined by $T e_{n}=w_{n} e_{n+1}$ for all $n$, where $\left\{e_{n}\right\}$ is an orthonormal basis (ONB, for short) of $\mathcal{H}$. If the index $n$ runs over the positive integers, then $T$ is called a unilateral weighted shift, while if $n$ runs over integers, then $T$ is called a bilateral weighted shift.

This work is mainly inspired by a recent paper [29], where complex symmetric weighted shifts are classified. In particular, it is proved that each complex symmetric unilateral weighted shift can be written as the direct sum of some finite-dimensional truncated weighted shifts with symmetric weights.

Definition 1.4. If $\left\{e_{i}\right\}_{i=1}^{n}$ is an ONB for some finite-dimensional Hilbert space, an operator of the form $T=\sum_{i=1}^{n-1} \lambda_{i}\left[e_{i+1} \otimes e_{i}\right]$ is called a finite-dimensional truncated weighted shift. In particular, if $\left|\lambda_{i}\right|=\left|\lambda_{n-i}\right|$ for each $1 \leq i \leq n-1$, then the weights $\left\{\lambda_{i}\right\}_{i=1}^{n-1}$ of $T$ are called symmetric.

Here the operator $e_{i+1} \otimes e_{i}$ is given by $e_{i+1} \otimes e_{i}(x)=\left\langle x, e_{i}\right\rangle e_{i+1}$ for $x$.

Noting that SSOs are intimately related to CSOs, one may wish to apply those methods developed in [29] to describe skew symmetric weighted shifts. Unfortunately, these methods are not applicable. One may see this in the proofs of Lemma 2.11 and Theorem 1.9. To obtain a complete characterization, we shall develop in this paper a completely different approach.

To state our main results, we need an extra notation.

Definition 1.5 ([1], page 95). Let $T \in \mathcal{B}(\mathcal{H})$. An operator $A \in \mathcal{B}(\mathcal{H})$ is called a transpose of $T$ if $A=C T^{*} C$ for some conjugation $C$ on $\mathcal{H}$.

If $T \in \mathcal{B}(\mathcal{H})$ is skew symmetric, then there exists a conjugation $C$ on $\mathcal{H}$ such that $-T=C T^{*} C$. It follows that $-T$ is a transpose of $T$. In general, an operator has more than one transpose [30, Ex. 2.2]. However, one can check that any two transposes of an operator are unitarily equivalent. We often write $T^{t}$ to denote 
a transpose of $T$. In general, there is no ambiguity especially when we write $T \cong T^{t}$. Here and in what follows, $\cong$ denotes unitary equivalence.

Definition $1.6([8])$. Let $T \in \mathcal{B}(\mathcal{H})$ and $\mathcal{M}$ be a nonzero subspace of $\mathcal{H}$. If $\mathcal{M}$ reduces $T$ and $\left.T\right|_{\mathcal{M}}$ is irreducible, then $\mathcal{M}$ is called a minimal reducing subspace of $T$. An operator is said to be completely reducible if it does not admit any minimal reducing subspace.

Note that a normal operator is completely reducible if and only if it has no eigenvalues.

Now we can list our results. The first result of this paper is the following result, which describes the block structure of SSOs.

Theorem 1.7. Let $T \in \mathcal{B}(\mathcal{H})$. Then $T$ is skew symmetric if and only if it is unitarily equivalent to a direct sum of (some of the summands may be absent)

(i) completely reducible SSOs,

(ii) irreducible $S S O s$, and

(iii) operators of the form $A \oplus\left(-A^{t}\right)$, where $A$ is irreducible and not skew symmetric.

Remark 1.8. (i) The above result decomposes a general SSO into the direct sum of three kinds of elementary ones. In Section 4, we shall construct several examples.

(ii) In a recent paper [18], Guo and the author provided a canonical decomposition of general CSOs. Theorem 1.7 is an analogue of this result for SSOs.

Based on Theorem 1.7, we give a concrete description of skew symmetric (unilateral or bilateral) weighted shifts. The following two results are main theorems of this paper.

Theorem 1.9. Let $T$ be a unilateral weighted shift on $\mathcal{H}$. Then $T$ is skew symmetric if and only if $T$ can be written as $T=\oplus_{i=1}^{\infty} T_{i}$, where each $T_{i}$ is a finitedimensional truncated weighted shift with symmetric weights and even rank.

Theorem 1.10. A bilateral weighted shift $T$ with weighted sequence $\left\{w_{i}\right\}_{i \in \mathbb{Z}}$ is skew symmetric if and only if exactly one of the following holds:

(i) $w_{i} \neq 0$ for all $i \in \mathbb{Z}$ and there exists $k \in \mathbb{Z}$ such that $\left|w_{i-1}\right|=\left|w_{2 k-i}\right|$ for all $i \in \mathbb{Z}$;

(ii) $T$ is an infinite direct sum of finite-dimensional truncated weighted shifts with symmetric weights and even rank;

(iii) $T$ is unitarily equivalent to an operator with the form $A \oplus A^{*} \oplus B$, where $A$ is an injective unilateral weighted shift and $B$ is absent or $B$ is a finite direct sum of finite-dimensional truncated weighted shifts with symmetric weights and even rank.

Remark 1.11. By the preceding two results and Theorems 3.1/4.1 of [29], each skew symmetric weighted shift is complex symmetric, while the converse does not hold in general. 
The rest of this paper is organized as follows. In Section 2, we shall give the proofs of Theorems 1.7 and 1.9. The proof of Theorem 1.10 is provided in Section 3. In Section 4, we shall give several illustrating examples of those three kinds of SSOs mentioned in Theorem 1.7.

\section{Proofs of Theorems 1.7 And 1.9}

This section is devoted to the proofs of Theorems 1.7 and 1.9. We first make some preparation. We remark that the proof of Theorem 1.7 follows a similar line as that of Theorem 1.6 in [18].

Lemma 2.1 ([30], Lem. 3.7). If $T \in \mathcal{B}(\mathcal{H})$, then $T \oplus\left(-T^{t}\right)$ is skew symmetric.

Corollary 2.2. Let $T \in \mathcal{B}(\mathcal{H})$ be a (unilateral or bilateral) weighted shift. Then $T \oplus T^{*}$ is skew symmetric.

Proof. We only give the proof in the case that $T$ is a unilateral weighted shift. The proof for the bilateral case is similar.

Assume that $T \in \mathcal{B}(\mathcal{H})$ is a unilateral weighted shift defined by $T e_{i}=\lambda_{i} e_{i+1}$ for $i \geq 1$, where $\left\{e_{i}\right\}_{i \geq 1}$ is an ONB of $\mathcal{H}$. Since $T$ is unitarily equivalent to the weighted shift with nonnegative weights $\left\{\left|\lambda_{i}\right|\right\}$, we may directly assume that $\lambda_{i} \geq 0$ for all $i$.

For $x \in \mathcal{H}$ with $x=\sum_{i} \alpha_{i} e_{i}$, define $C x=\sum_{i} \overline{\alpha_{i}}(-1)^{i} e_{i}$. Then one can check that $C$ is a conjugation on $\mathcal{H}$ and, for each $i \geq 1$,

$$
C T C e_{i}=(-1)^{i} C T e_{i}=(-1)^{i} \lambda_{i} C e_{i+1}=(-1)^{2 i+1} \lambda_{i} e_{i+1}=-T e_{i} .
$$

So $-T=C T C$ and $-T^{*}=C T^{*} C$, which means that $-T^{*}$ is a transpose of $T$. By Lemma 2.1, $T \oplus T^{*}$ is skew symmetric.

The following result proved in [18, Lem. 2.2] is very useful. For the reader's convenience we still present it here.

Lemma 2.3. Let $T \in \mathcal{B}(\mathcal{H})$ and $A=C T^{*} C^{-1}$, where $C$ is an antiunitary operator on $\mathcal{H}$. If $\mathcal{M}$ is a reducing subspace of $T$, then $C(\mathcal{M})$ is a reducing subspace of $A$ and $\left.A\right|_{C(\mathcal{M})} \cong\left(\left.T\right|_{\mathcal{M}}\right)^{t}$. In particular, $\left.T\right|_{\mathcal{M}}$ is irreducible if and only if $\left.A\right|_{C(\mathcal{M})}$ is irreducible.

Proof. Denote $\mathcal{N}=C(\mathcal{M})$. It is easy to check that $\mathcal{N}$ is a reducing subspace of $A$. For $x \in \mathcal{M}$, define $D x=C x$. Thus $D: \mathcal{M} \rightarrow \mathcal{N}$ is an antiunitary operator. Since $A C=C T^{*}$, we obtain $\left(\left.A\right|_{\mathcal{N}}\right)\left(\left.C\right|_{\mathcal{M}}\right)=\left(\left.C\right|_{\mathcal{M}}\right)\left(\left.T^{*}\right|_{\mathcal{M}}\right)$, that is, $\left(\left.A\right|_{\mathcal{N}}\right) D=D\left(\left.T^{*}\right|_{\mathcal{M}}\right)$. Arbitrarily choose a conjugation $E$ on $\mathcal{M}$. Then we have

$$
\begin{aligned}
\left.A\right|_{\mathcal{N}}=D\left(\left.T^{*}\right|_{\mathcal{M}}\right) D^{-1} & =(D E)\left[E\left(\left.T^{*}\right|_{\mathcal{M}}\right) E\right]\left(E D^{-1}\right) \\
& =(D E)\left[E\left(\left.T\right|_{\mathcal{M}}\right)^{*} E\right]\left(E D^{-1}\right) .
\end{aligned}
$$

Noting that $D E: \mathcal{M} \rightarrow \mathcal{N}$ is unitary and $(D E)^{-1}=E D^{-1}$, it follows that $\left.A\right|_{\mathcal{N}} \cong$ $\left(\left.T\right|_{\mathcal{M}}\right)^{t}$. The assertion about minimal reducing subspace follows readily.

Given $T \in \mathcal{B}(\mathcal{H})$ and a cardinal number $n, 1 \leq n \leq \aleph_{0}$, we let $\mathcal{H}^{(n)}$ denote the direct sum of $n$ copies of $\mathcal{H}$ and let $T^{(n)}$ denote the direct sum of $n$ copies of $T$, acting on $\mathcal{H}^{(n)}$ (see [6, Def. 6.3]). 
Proposition 2.4 ([18], Prop. 2.3). Let $T \in \mathcal{B}(\mathcal{H})$ and $T=T_{0} \oplus\left(\oplus_{i \in \Lambda} T_{i}^{\left(n_{i}\right)}\right)$, where $T_{0}$ is completely reducible, $T_{i}$ is irreducible and $1 \leq n_{i} \leq \infty$ for $i \in \Lambda$; moreover, $T_{i} ¥ T_{j}$ whenever $i, j \in \Lambda$ and $i \neq j$. Then each reducing subspace $\mathcal{M}$ of $T$ has the form of $\mathcal{M}_{0} \oplus\left(\oplus_{i \in \Lambda} \mathcal{M}_{i}\right)$, where $\mathcal{M}_{0}$ is a reducing subspace of $T_{0}$ and $\mathcal{M}_{i}$ is a reducing subspace of $T_{i}^{\left(n_{i}\right)}$ for $i \in \Lambda$.

Corollary 2.5. Let $T \in \mathcal{B}(\mathcal{H})$ and $T=T_{0} \oplus\left(\oplus_{i \in \Lambda} T_{i}^{\left(n_{i}\right)}\right)$, where $T_{0} \in \mathcal{B}\left(\mathcal{H}_{0}\right)$ is completely reducible, $T_{i} \in \mathcal{B}\left(\mathcal{H}_{i}\right)$ is irreducible and $1 \leq n_{i} \leq \infty$ for $i \in \Lambda$; moreover, $T_{i} \nsucceq T_{j}$ whenever $i, j \in \Lambda$ and $i \neq j$. Let $\mathcal{M}$ be a nonzero subspace of $\mathcal{H}$. Then $\mathcal{M}$ is a minimal reducing subspace of $T$ if and only if there exists $i \in \Lambda$ such that $\mathcal{M} \subset \mathcal{H}_{i}^{\left(n_{i}\right)}$ and $\mathcal{M}$ is a minimal reducing subspace of $T_{i}^{\left(n_{i}\right)}$.

Corollary 2.6. Let $T \in \mathcal{B}(\mathcal{H})$ and $T=T_{0} \oplus\left(\oplus_{i \in \Lambda} T_{i}^{\left(n_{i}\right)}\right)$, where $T_{0} \in \mathcal{B}\left(\mathcal{H}_{0}\right)$ is completely reducible, $T_{i} \in \mathcal{B}\left(\mathcal{H}_{i}\right)$ is irreducible and $1 \leq n_{i} \leq \infty$ for $i \in \Lambda$; moreover, $T_{i} \not T_{j}$ whenever $i, j \in \Lambda$ and $i \neq j$. If $T$ is skew symmetric, then $T_{0}$ and $\oplus_{i \in \Lambda} T_{i}^{\left(n_{i}\right)}$ are both skew symmetric.

Proof. Since $T$ is skew symmetric, there is a conjugation $C$ on $\mathcal{H}$ such that $C T C=-T^{*}$. By Lemma 2.3, $C$ maps one minimal reducing subspace of $T$ to another. It follows from Corollary 2.5 that $C\left(\oplus_{i \in \Lambda} \mathcal{H}_{i}^{\left(n_{i}\right)}\right) \subset \oplus_{i \in \Lambda} \mathcal{H}_{i}^{\left(n_{i}\right)}$. Since $C$ is a conjugation and $C^{2}=I$, one can see that $C\left(\oplus_{i \in \Lambda} \mathcal{H}_{i}^{\left(n_{i}\right)}\right)=\oplus_{i \in \Lambda} \mathcal{H}_{i}^{\left(n_{i}\right)}$ and hence $C\left(\mathcal{H}_{0}\right)=\mathcal{H}_{0}$.

Set $C_{1}=\left.C\right|_{\mathcal{H}_{0}}$ and $C_{2}=\left.C\right|_{\mathcal{H}_{0}^{\perp}}$. Then $C_{1}, C_{2}$ are conjugations and $C=C_{1} \oplus C_{2}$. It follows from $-T^{*}=C T C$ that $-T_{0}^{*}=C_{1} T_{0} C_{1}$ and

$$
-\left(\oplus_{i \in \Lambda} T_{i}^{\left(n_{i}\right)}\right)^{*}=C_{2}\left(\oplus_{i \in \Lambda} T_{i}^{\left(n_{i}\right)}\right) C_{2} \text {. }
$$

This completes the proof.

If $A \in \mathcal{B}(\mathcal{H})$ is irreducible, then the commutant algebra $\left\{A, A^{*}\right\}^{\prime}$ of $\left\{A, A^{*}\right\}$ equals $\mathbb{C} I$; whence the following result is clear. The reader is also referred to $[17$, Prop. 7.4] for a proof.

Lemma 2.7. Let $T=A^{(n)}$, where $A \in \mathcal{B}(\mathcal{H})$ is irreducible and $1 \leq n \leq \infty$. If $\mathcal{M}$ is a nonzero reducing subspace of $T$, then the following are equivalent:

(i) $\left.T\right|_{\mathcal{M}} \cong A$;

(ii) $\left.T\right|_{\mathcal{M}}$ is irreducible;

(iii) There exist complex numbers $\left\{\alpha_{i}\right\}_{i=1}^{n}$ with $0<\sum_{i=1}^{n}\left|\alpha_{i}\right|^{2}<\infty$ such that

$$
\mathcal{M}=\left\{\oplus_{i=1}^{n} \alpha_{i} \xi: \xi \in \mathcal{H}\right\} .
$$

Corollary 2.8. Let $T \in \mathcal{B}(\mathcal{H})$ and $T=T_{0} \oplus\left(\oplus_{i \in \Lambda} T_{i}^{\left(n_{i}\right)}\right)$, where $T_{0} \in \mathcal{B}\left(\mathcal{H}_{0}\right)$ is completely reducible, $T_{i} \in \mathcal{B}\left(\mathcal{H}_{i}\right)$ is irreducible and $1 \leq n_{i} \leq \infty$ for $i \in \Lambda$; moreover, $T_{i} \not T_{j}$ whenever $i, j \in \Lambda$ and $i \neq j$. If $\mathcal{M}$ is a minimal reducing subspace of $T$, then $\left.T\right|_{\mathcal{M}} \cong T_{i}$ for some $i \in \Lambda$.

Proposition 2.9. Let $T=A^{(n)}$, where $A \in \mathcal{B}(\mathcal{H})$ is irreducible and $1 \leq n \leq \infty$. Then $T$ is skew symmetric if and only if exactly one of the following holds: 
(i) $A$ is skew symmetric;

(ii) $n \in\{2 i: i \in \mathbb{N}\} \cup\{\infty\}, A \cong\left(-A^{t}\right)$ and $A$ is not skew symmetric.

Proof. "£". When $A$ is skew symmetric, the sufficiency is trivial. Now we assume that statement (ii) holds. By the hypothesis, we have $A \cong\left(-A^{t}\right)$. Thus

$$
T=A^{(n)}=(A \oplus A)^{\left(\frac{n}{2}\right)} \cong\left(A \oplus\left(-A^{t}\right)\right)^{\left(\frac{n}{2}\right)} .
$$

It follows from Lemma 2.1 that $T$ is skew symmetric.

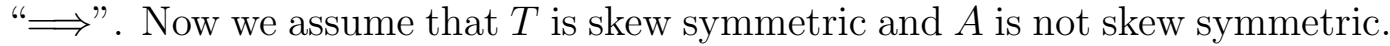
Then we need only prove that $A \cong\left(-A^{t}\right)$ and $n$ is not odd. We need only give the proof in the case that $n<\infty$, the proof for $n=\infty$ is easier.

For convenience, we write

$$
T^{(n)}=\left[\begin{array}{llll}
A & & & \\
& A & & \\
& & \ddots & \\
& & & A
\end{array}\right] \begin{gathered}
\mathcal{H}_{1} \\
\mathcal{H}_{2} \\
\vdots \\
\mathcal{H}_{n}
\end{gathered},
$$

where $\mathcal{H}_{1}=\mathcal{H}_{2}=\cdots \mathcal{H}_{n}=\mathcal{H}$.

We shall proceed by induction on $n$.

If $n \leq 2$, then we claim that $n=2$. Otherwise, $n=1$ and $A=T$ is skew symmetric, contradicting the hypothesis.

Now suppose we have proved that $n \leq k$ implies $n$ is even. Now assume that $n=k+1$. Since $T$ is skew symmetric, there is a conjugation $C$ on $\mathcal{H}^{(n)}$ such that $-T=C T^{*} C$. Denote $\mathcal{N}=C\left(\mathcal{H}_{1}\right)$. Since $A=\left.T\right|_{\mathcal{H}_{1}}$ is irreducible and $-T=C T^{*} C$, it follows from Lemma 2.3 that $\mathcal{N}$ is a minimal reducing subspace of $T$ and $\left(-\left.T\right|_{\mathcal{N}}\right) \cong\left(\left.T\right|_{\mathcal{H}_{1}}\right)^{t}=A^{t}$; whence $\left.T\right|_{\mathcal{N}}$ is irreducible. By Lemma 2.7 , this implies $\left.T\right|_{\mathcal{N}} \cong A$. Therefore we obtain $A \cong\left(-A^{t}\right)$. Now it remains to prove that $n$ is even.

Since $\mathcal{N}=C\left(\mathcal{H}_{1}\right)$ and $\mathcal{H}_{1}=C(\mathcal{N})$, we obtain $C\left(\vee\left\{\mathcal{H}_{1}, \mathcal{N}\right\}\right) \subset \vee\left\{\mathcal{H}_{1}, \mathcal{N}\right\}$. Here $\vee$ denotes closed linear span. Furthermore, $C\left(\vee\left\{\mathcal{H}_{1}, \mathcal{N}\right\}\right)=\vee\left\{\mathcal{H}_{1}, \mathcal{N}\right\}$ and $C\left(\left\{\mathcal{H}_{1}, \mathcal{N}\right\}^{\perp}\right)=\left\{\mathcal{H}_{1}, \mathcal{N}\right\}^{\perp}$. So $\vee\left\{\mathcal{H}_{1}, \mathcal{N}\right\}$ and $\left\{\mathcal{H}_{1}, \mathcal{N}\right\}^{\perp}$ both reduce $C$.

On the other hand, Since $\mathcal{N}$ is irreducible, it follows from Lemma 2.7 that

$$
\mathcal{N}=\left\{\oplus_{i=1}^{n} \alpha_{i} x: x \in \mathcal{H}\right\}
$$

for some nonzero $\left(\alpha_{1}, \cdots, \alpha_{n}\right) \in \mathbb{C}^{n}$. We claim that $\left(\alpha_{2}, \alpha_{3}, \cdots, \alpha_{n}\right) \neq 0$. In fact, if not, then $\mathcal{H}_{1}=\mathcal{N}$ and $C\left(\mathcal{H}_{1}\right)=\mathcal{H}_{1}$. It follows that $A=\left.T\right|_{\mathcal{H}_{1}}$ is skew symmetric, a contradiction. Denote $\mathcal{M}=\left\{\oplus_{i=2}^{n} \alpha_{i} x: x \in \mathcal{H}\right\}$. Then $\mathcal{M} \neq\{0\}$, and it follows from Lemma 2.7 that $\mathcal{M}$ reduces $T$ and $\left.T\right|_{\mathcal{M}} \cong A$. Noting that

$$
\vee\left\{\mathcal{H}_{1}, \mathcal{N}\right\}=\mathcal{H}_{1} \oplus \mathcal{M}
$$

$\vee\left\{\mathcal{H}_{1}, \mathcal{N}\right\}$ reduces $T$ and $\left.T\right|_{\vee\left\{\mathcal{H}_{1}, \mathcal{N}\right\}}=\left(\left.T\right|_{\mathcal{M}}\right) \oplus\left(\left.T\right|_{\mathcal{H}_{1}}\right) \cong A^{(2)}$. It follows that $\left\{\mathcal{H}_{1}, \mathcal{N}\right\}^{\perp}$ reduces $T$. Since $\left\{\mathcal{H}_{1}, \mathcal{N}\right\}^{\perp}$ also reduces $C$, we deduce that $\left.T\right|_{\left\{\mathcal{H}_{1}, \mathcal{N}\right\}^{\perp}}$ is skew symmetric. By [17, Cor. 7.5], there exists $m \leq n$ such that $\left.T\right|_{\left\{\mathcal{H}_{1}, \mathcal{N}\right\}^{\perp}} \cong$ $A^{(m)}$.

Note that if $A \in \mathcal{B}(\mathcal{H})$ is irreducible, then the commutant algebra $\left\{A, A^{*}\right\}^{\prime}$ of $\left\{A, A^{*}\right\}$ equals $\mathbb{C} I$; hence the commutant algebra of $\left\{A^{(n)},\left(A^{*}\right)^{(n)}\right\}$ is $*$-isomorphic 
to the matrix algebra $M_{n}(\mathbb{C})$. It follows that $m=n-2$ and $\left.T\right|_{\left\{\mathcal{H}_{1}, \mathcal{N}\right\}^{\perp}} \cong A^{(n-2)}$. Noting that $n-2 \leq k$ and $\left.T\right|_{\left\{\mathcal{H}_{1}, \mathcal{N}\right\}^{\perp}}$ is skew symmetric, by the induction hypothesis, we deduce that $n-2$ is even, and so is $n$. This completes the proof.

Lemma 2.10 ([8], Prop. 2.4). If $T \in \mathcal{B}(\mathcal{H})$, then $T$ admits the decomposition $T=T_{0} \oplus\left(\bigoplus_{i \in \Lambda} T_{i}\right)$, where $T_{0} \in \mathcal{B}\left(\mathcal{H}_{0}\right)$ is completely reducible and $T_{i} \in \mathcal{B}\left(\mathcal{H}_{i}\right)$ is irreducible for all $i \in \Lambda$.

Proof of Theorem 1.7. By Lemma 2.1, the sufficiency is obvious. It suffices to prove the necessity.

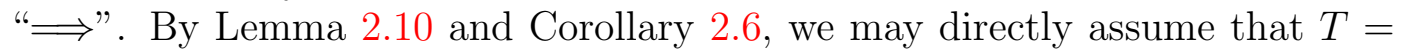
$\oplus_{i=1}^{\infty} T_{i}^{\left(n_{i}\right)}$, where $T_{i} \in \mathcal{B}\left(\mathcal{H}_{i}\right)$ is irreducible and $1 \leq n_{i} \leq \infty$ for $i \geq 1$; moreover, $T_{i} \supsetneqq T_{j}$ whenever $i \neq j$. Thus $\mathcal{H}=\oplus_{i \geq 1} \mathcal{H}_{i}^{\left(n_{i}\right)}$. To be convenient, for each $i \geq 1$, we write

where $\mathcal{H}_{i, 1}=\mathcal{H}_{i, 2}=\cdots=\mathcal{H}_{i, n_{i}}=\mathcal{H}_{i}$.

$$
T_{i}^{\left(n_{i}\right)}=\left[\begin{array}{llll}
T_{i} & & & \\
& T_{i} & & \\
& & \ddots & \\
& & & T_{i}
\end{array}\right] \begin{gathered}
\mathcal{H}_{i, 1} \\
\mathcal{H}_{i, 2} \\
\vdots \\
\mathcal{H}_{i, n_{i}}
\end{gathered}
$$

Since $T$ is an SSO, there exists a conjugation $C$ on $\mathcal{H}$ such that $-T=C T^{*} C$. For each $i \geq 1$, denote $\mathcal{M}_{i}=C\left(\mathcal{H}_{i, 1}\right)$. Since $T_{i}=\left.T\right|_{\mathcal{H}_{i}}$ is irreducible, it follows from Lemma 2.3 that $\mathcal{M}_{i}$ reduces $T,\left.T\right|_{\mathcal{M}_{i}}$ is irreducible and $\left(-\left.T\right|_{\mathcal{M}_{i}}\right) \cong T_{i}^{t}$. By Corollary 2.5, there exists unique $\tau_{i} \in \mathbb{N}$ such that $\mathcal{M}_{i} \subset \mathcal{H}_{\tau_{i}}^{\left(n_{\tau_{i}}\right)}$. This defines a map $\tau$ on $\mathbb{N}$. It follows from Lemma 2.7 that $\left.T\right|_{\mathcal{M}_{i}}=\left.T_{\tau_{i}}^{\left(n_{\tau_{i}}\right)}\right|_{\mathcal{M}_{i}} \cong T_{\tau_{i}}$. Then we obtain

$$
\left(-T_{\tau_{i}}\right) \cong T_{i}^{t}, \quad \forall i \geq 1
$$

Claim 1. For each $i \geq 1$, if $\mathcal{N} \subset \mathcal{H}_{i}^{\left(n_{i}\right)}$ is a minimal reducing subspace of $T$, then $C(\mathcal{N}) \subset \mathcal{H}_{\tau_{i}}^{\left(n_{\tau_{i}}\right)}$.

Since $\left.T\right|_{\mathcal{N}}$ is irreducible, it follows from Lemmas 2.3 and 2.7 that $\left.T\right|_{C(\mathcal{N})}$ is irreducible and

$$
\left(-\left.T\right|_{C(\mathcal{N})}\right) \cong\left(\left.T\right|_{\mathcal{N}}\right)^{t}=\left(T_{i}\right)^{t}
$$

Moreover there exists $j \in \Lambda$ such that $C(\mathcal{N}) \subset \mathcal{H}_{j}^{\left(n_{j}\right)}$. Thus $\left.T\right|_{C(\mathcal{N})} \cong T_{j}$. In view of $(2.1)$ and $(2.2)$, we obtain $\left(-T_{j}\right) \cong\left(T_{i}\right)^{t} \cong\left(-T_{\tau_{i}}\right)$. By the hypothesis, it follows that $j=\tau_{i}$. This proves the claim.

Claim 2. $C\left(\mathcal{H}_{i}^{\left(n_{i}\right)}\right)=\mathcal{H}_{\tau_{i}}^{\left(n_{\tau_{i}}\right)}$ for any $i \geq 1$.

Fix an $i \geq 1$ and denote $j=\tau_{i}$. In view of (2.1), we have $\left(-T_{\tau_{i}}\right) \cong T_{i}^{t}$ and $\left(-T_{\tau_{j}}\right) \cong T_{j}^{t}$. It follows that $T_{\tau_{j}}^{t} \cong\left(-T_{j}\right)$ and $T_{\tau_{j}} \cong T_{i}$. By the hypothesis on the decomposition $T=\oplus_{i \geq 1} T_{i}^{\left(n_{i}\right)}$, one can deduce that $i=\tau_{j}$. Hence $\tau^{2}(i)=i$. It follows immediately from Claim 1 that

$$
C\left(\mathcal{H}_{i}^{\left(n_{i}\right)}\right) \subset \mathcal{H}_{\tau_{i}}^{\left(n_{\tau_{i}}\right)} \text { and } C\left(\mathcal{H}_{\tau_{i}}^{\left(n_{\tau_{i}}\right)}\right) \subset \mathcal{H}_{i}^{\left(n_{i}\right)} .
$$

Since $C^{-1}=C$, we have

$$
C\left(\mathcal{H}_{i}^{\left(n_{i}\right)}\right) \subset \mathcal{H}_{\tau_{i}}^{\left(n_{\tau_{i}}\right)} \subset C\left(\mathcal{H}_{i}^{\left(n_{i}\right)}\right)
$$


that is, $C\left(\mathcal{H}_{i}^{\left(n_{i}\right)}\right)=\mathcal{H}_{\tau_{i}}^{\left(n_{\tau_{i}}\right)}$. This proves Claim 2 .

By the above argument, the map $\tau: i \mapsto \tau_{i}$ is invertible and $\tau^{-1}=\tau$. Thus $\tau$ induces the following partition of $\mathbb{N}$

$$
\left\{\left\{i, \tau_{i}\right\}: i \geq 1\right\}
$$

denoted by $\left\{\Lambda_{r}: r \in \Gamma\right\}$. Then $\cup_{r \in \Gamma} \Lambda_{r}=\mathbb{N}$ and $1 \leq$ card $\Lambda_{r} \leq 2$ for all $r \in \Gamma$. Thus $T$ can be written as

$$
T=\bigoplus_{r \in \Gamma}\left(\oplus_{i \in \Lambda_{r}} T_{i}^{\left(n_{i}\right)}\right)
$$

with respect to the decomposition

$$
\mathcal{H}=\bigoplus_{r \in \Gamma}\left(\oplus_{i \in \Lambda_{r}} \mathcal{H}_{i}^{\left(n_{i}\right)}\right)
$$

Noting that $C\left(\oplus_{i \in \Lambda_{r}} \mathcal{H}_{i}^{\left(n_{i}\right)}\right)=\oplus_{i \in \Lambda_{r}} \mathcal{H}_{i}^{\left(n_{i}\right)}$ for all $r \in \Gamma$, it follows that $\oplus_{i \in \Lambda_{r}} T_{i}^{\left(n_{i}\right)}$ is skew symmetric for all $r \in \Gamma$. So, in order to complete the proof, it suffices to prove that each $\oplus_{i \in \Lambda_{r}} T_{i}^{\left(n_{i}\right)}$ admits the desired decomposition for $r \in \Gamma$.

Claim 3. $n_{i}=n_{\tau_{i}}$ for all $i \geq 1$.

Now fix an $i \geq 1$. For each $1 \leq j \leq n_{i}$, denote $\mathcal{N}_{j}=C\left(\mathcal{H}_{i, j}\right)$. Then, by Claim $2, \oplus_{j=1}^{n_{i}} \mathcal{N}_{j}=C\left(\mathcal{H}_{i}^{\left(n_{i}\right)}\right)=\mathcal{H}_{\tau_{i}}^{\left(n_{\tau_{i}}\right)}$. Hence it follows from Lemma 2.7 that

$$
T_{\tau_{i}}^{\left(n_{\tau_{i}}\right)}=\left.T\right|_{\mathcal{H}_{\tau_{i}}^{\left(n_{\tau_{i}}\right)}}=\bigoplus_{j=1}^{n_{i}}\left(\left.T\right|_{\mathcal{N}_{j}}\right) \cong T_{\tau_{i}}^{\left(n_{i}\right)} .
$$

Since $T_{\tau_{i}}$ is irreducible, by comparing commutant algebras, one can see that $n_{i}=n_{\tau_{i}}$. This proves Claim 3 .

Now we are going to conclude our proof. Fix an $r \in \Gamma$.

If card $\Lambda_{r}=1$ and $k \in \Lambda_{r}$, then $\oplus_{i \in \Lambda_{r}} T_{i}^{\left(n_{i}\right)}=T_{k}^{\left(n_{k}\right)}$. Since $T_{k}^{\left(n_{k}\right)}$ is an SSO, by Proposition 2.9, it admits the desired decomposition.

If card $\Lambda_{r}=2$ and $k \in \Lambda_{r}$, then $k \neq \tau_{k}$ and $\oplus_{i \in \Lambda_{r}} T_{i}^{\left(n_{i}\right)}=T_{k}^{\left(n_{k}\right)} \oplus T_{\tau_{k}}^{\left(n_{\tau_{k}}\right)}$. In view of Claim 3 and (2.1), we have

$$
T_{k}^{\left(n_{k}\right)} \oplus T_{\tau_{k}}^{\left(n_{\tau_{k}}\right)}=T_{k}^{\left(n_{k}\right)} \oplus T_{\tau_{k}}^{\left(n_{k}\right)} \cong\left(T_{k}\right)^{\left(n_{k}\right)} \oplus\left(-T_{k}^{t}\right)^{\left(n_{k}\right)}=\left(T_{k} \oplus\left(-T_{k}^{t}\right)\right)^{\left(n_{k}\right)} .
$$

We claim that $T_{k}$ is not skew symmetric. In fact, if not, then $T_{k} \cong\left(-T_{k}^{t}\right)$. In view of $(2.1)$, we have $T_{k} \cong T_{\tau_{k}}$. This contradicts the fact that $T_{k} \nsucceq T_{\tau_{k}}$ since $k \neq \tau_{k}$. Therefore $\oplus_{i \in \Lambda_{r}} T_{i}^{\left(n_{i}\right)}$ admits the desired decomposition. This completes the proof.

Lemma 2.11. Let $n \in \mathbb{N}$ and $\left\{e_{i}\right\}_{i=1}^{n}$ be an ONB of $\mathbb{C}^{n}$. Assume that

$$
T=\sum_{i=1}^{n-1} \lambda_{i} e_{i+1} \otimes e_{i},
$$

where $\lambda_{i} \neq 0$ for all $1 \leq i \leq n-1$. Then $T$ is skew symmetric if and only if $n$ is odd and $\left|\lambda_{i}\right|=\left|\lambda_{n-i}\right|$ for all $1 \leq i \leq n-1$. 
Proof. Since $T \cong \sum_{i=1}^{n-1}\left|\lambda_{i}\right| e_{i+1} \otimes e_{i}$, we may directly assume that $\lambda_{i}>0$ for $1 \leq i \leq n-1$. Then it is obvious that $T^{*}=\sum_{i=1}^{n-1} \lambda_{i} e_{i} \otimes e_{i+1}$.

"£". For $x \in \mathbb{C}^{n}$ with $x=\sum_{i=1}^{n} \alpha_{i} e_{i}$, define

$$
C x=\sum_{i=1}^{n} \overline{\alpha_{i}}(-1)^{i-\frac{n+1}{2}} e_{n-i+1} .
$$

It is easy to see that $C$ is invertible, conjugate-linear and isometric. Moreover, one can check that

$$
\begin{aligned}
C^{2} x & =C\left(\sum_{i=1}^{n} \overline{\alpha_{i}}(-1)^{i-\frac{n+1}{2}} e_{n-i+1}\right) \\
& =\sum_{i=1}^{n} \alpha_{i}(-1)^{i-\frac{n+1}{2}} C e_{n-i+1} \\
& =\sum_{i=1}^{n} \alpha_{i}(-1)^{i-\frac{n+1}{2}}(-1)^{\frac{n+1}{2}-i} e_{i}=x .
\end{aligned}
$$

Thus $C^{-1}=C$, and hence $C$ is a conjugation on $\mathbb{C}^{n}$.

For $1 \leq i \leq n-1$, one can check that

$$
C T e_{i}=C \lambda_{i} e_{i+1}=\lambda_{i}(-1)^{i+1-\frac{n+1}{2}} e_{n-i}
$$

and

$$
-T^{*} C e_{i}=-(-1)^{i-\frac{n+1}{2}} T^{*} e_{n-i+1}=(-1)^{i+1-\frac{n+1}{2}} \lambda_{n-i} e_{n-i} ;
$$

that is, $C T e_{i}=-T^{*} C e_{i}$. On the other hand, $C T e_{n}=0=-(-1)^{n-\frac{n+1}{2}} T^{*} e_{1}=$ $-T^{*} C e_{n}$. It follows that $C T=-T^{*} C$ and $T$ is skew symmetric.

"ב". Assume that $C$ is a conjugation on $\mathbb{C}^{n}$ and $C T C=-T^{*}$. Thus $C T^{i} C=$ $(-1)^{i}\left(T^{*}\right)^{i}$ for $i \geq 1$. It follows that $C\left(\operatorname{ker} T^{i}\right)=\operatorname{ker}\left(T^{*}\right)^{i}$ for all $i \geq 1$. Note that $\operatorname{ker} T^{i}=\vee\left\{e_{j}: n-i+1 \leq j \leq n\right\}$ and $\operatorname{ker}\left(T^{*}\right)^{i}=\vee\left\{e_{j}: 1 \leq j \leq i\right\}$ for each $1 \leq i \leq n$. Since $\langle C x, C y\rangle=\langle y, x\rangle$ for all $x, y \in \mathbb{C}^{n}$, there exist complex numbers $\left\{\mu_{i}\right\}_{i=1}^{n}$ with $\left|\mu_{i}\right|=1$ such that $C e_{i}=\mu_{i} e_{n-i+1}$ for any $1 \leq i \leq n$.

Fix an $i$ with $1 \leq i \leq n$. We have

$$
\begin{aligned}
(-1)^{i} \mu_{1} \lambda_{n-i+1} \cdots \lambda_{n-1} \lambda_{n} e_{n-i} & =(-1)^{i} \mu_{1}\left(T^{*}\right)^{i} e_{n}=(-1)^{i}\left(T^{*}\right)^{i} C e_{1} \\
& =C T^{i} e_{1}=C\left(\lambda_{1} \lambda_{2} \cdots \lambda_{i} e_{i+1}\right) \\
& =\lambda_{1} \lambda_{2} \cdots \lambda_{i} \mu_{i+1} e_{n-i} .
\end{aligned}
$$

It follows that $\lambda_{1} \lambda_{2} \cdots \lambda_{i}=\lambda_{n-i+1} \cdots \lambda_{n-1} \lambda_{n}$. Since $1 \leq i \leq n$ is arbitrary, it follows that $\lambda_{i}=\lambda_{n-i+1}$ for $1 \leq i \leq n-1$.

Now it remains to prove that $n$ is odd. If not, then $n$ is even. Set $k=\frac{n}{2}$. Then $C e_{k}=\mu_{k} e_{k+1}$ and hence $C e_{k+1}=\mu_{k} e_{k}$. A direct calculation shows that

$$
-\mu_{k} \lambda_{k} e_{k}=-T^{*} \mu_{k} e_{k+1}=-T^{*} C e_{k}=C T e_{k}=C \lambda_{k} e_{k+1}=\lambda_{k} \mu_{k} e_{k},
$$

which implies that $\lambda_{k} \mu_{k}=0$, a contradiction. This completes the proof.

By the proof for the sufficiency of Lemma 2.11, the following corollary is clear. 
Corollary 2.12. Let $n \in \mathbb{N}$ and $\left\{e_{i}\right\}_{i=1}^{n}$ be an ONB of $\mathbb{C}^{n}$. Assume that

$$
T=\sum_{i=1}^{n-1} \lambda_{i} e_{i+1} \otimes e_{i}
$$

If $n$ is odd and $\left|\lambda_{i}\right|=\left|\lambda_{n-i}\right|$ for all $1 \leq i \leq n-1$, then $T$ is skew symmetric.

Now we can give the proof of Theorem 1.9.

Proof of Theorem 1.9. "£". It suffices to prove that each $T_{i}$ is skew symmetric.

Now fix an $i$. Assume that

$$
T_{i}=\sum_{i=1}^{n-1} \lambda_{i} e_{i+1} \otimes e_{i}
$$

where $\left\{e_{i}\right\}_{i=1}^{n}$ is an ONB of the underlying space of $T_{i},\left|\lambda_{j}\right|=\left|\lambda_{n-j}\right|$ for all $1 \leq$ $j \leq n-1$ and $\operatorname{rank} T_{i}$ is even. Noting that $T_{i} \cong \sum_{j=1}^{n-1}\left|\lambda_{j}\right| e_{j+1} \otimes e_{j}$, we may directly assume that $\lambda_{j} \geq 0$ for each $j$.

If $n$ is odd, then the result follows immediately from Corollary 2.12.

Now we assume that $n$ is even. Denote $k=\frac{n}{2}$. First we claim that $\lambda_{k}=0$. In fact, if not, then

$$
\begin{aligned}
\operatorname{rank} T_{i} & =\operatorname{card}\left\{1 \leq j \leq n-1: \lambda_{j} \neq 0\right\} \\
& =1+2 \cdot \operatorname{card}\left\{1 \leq j \leq k-1: \lambda_{j} \neq 0\right\}
\end{aligned}
$$

is odd, a contradiction.

For each $x=\sum_{j=1}^{n} \alpha_{j} e_{j}$, define

$$
C x=\sum_{j=1}^{k} \overline{\alpha_{j}}(-1)^{j} e_{n-j+1}+\sum_{j=k+1}^{n} \overline{\alpha_{j}}(-1)^{j+1} e_{n-j+1} .
$$

Then one can check that $C$ is conjugate-linear and isometric. Moreover, one can see that $C e_{j}=(-1)^{j} e_{n-j+1}$ for $1 \leq j \leq k$ and $C e_{j}=(-1)^{j+1} e_{n-j+1}$ for $k+1 \leq j \leq n$. Then for $x=\sum_{j=1}^{n} \alpha_{j} e_{j}$ we have

$$
\begin{aligned}
C^{2} x & =C\left(\sum_{j=1}^{k} \overline{\alpha_{j}}(-1)^{j} e_{n-j+1}+\sum_{j=k+1}^{n} \overline{\alpha_{j}}(-1)^{j+1} e_{n-j+1}\right) \\
& =\sum_{j=1}^{k} \alpha_{j}(-1)^{j} C e_{n-j+1}+\sum_{j=k+1}^{n} \alpha_{j}(-1)^{j+1} C e_{n-j+1} \\
& =\sum_{j=1}^{k} \alpha_{j}(-1)^{j}(-1)^{n-j+2} e_{j}+\sum_{j=k+1}^{n} \alpha_{j}(-1)^{j+1}(-1)^{n-j+1} e_{j}=x .
\end{aligned}
$$

Hence $C$ is invertible and $C^{-1}=C$. It follows that $C$ is a conjugation on the underlying space of $T_{i}$. Now it remains to check that $C T_{i}=-T_{i}^{*} C$. 
If $1 \leq j<k$, then

$$
\begin{aligned}
C T_{i} e_{j} & =C \lambda_{j} e_{j+1}=\lambda_{j} C e_{j+1}=(-1)^{j+1} \lambda_{j} e_{n-j} \\
& =(-1)^{j+1} \lambda_{n-j} e_{n-j}=(-1)^{j+1} T_{i}^{*} e_{n-j+1} \\
& =-T_{i}^{*}(-1)^{j} e_{n-j+1}=-T_{i}^{*} C e_{j} .
\end{aligned}
$$

If $k+1 \leq j<n$, then

$$
\begin{aligned}
C T_{i} e_{j} & =C \lambda_{j} e_{j+1}=\lambda_{j} C e_{j+1}=(-1)^{j+2} \lambda_{j} e_{n-j} \\
& =(-1)^{j+2} \lambda_{n-j} e_{n-j}=(-1)^{j+2} T_{i}^{*} e_{n-j+1} \\
& =-T_{i}^{*}(-1)^{j+1} e_{n-j+1}=-T_{i}^{*} C e_{j} .
\end{aligned}
$$

Also one can check that

$$
C T_{i} e_{k}=C \lambda_{k} e_{k+1}=0=(-1)^{k+1} \lambda_{k} e_{k}=-T_{i}^{*}(-1)^{k} e_{k+1}=-T_{i}^{*} C e_{k}
$$

and

$$
C T_{i} e_{n}=0=(-1)^{n+2} T_{i}^{*} e_{1}=-T_{i}^{*}(-1)^{n+1} e_{1}=-T_{i}^{*} C e_{n} .
$$

Thus we have checked that $C T_{i} e_{j}=-T_{i}^{*} C e_{j}$ for all $1 \leq j \leq n$. Hence $C T_{i}=$ $-T_{i}^{*} C$ and $T_{i}$ is skew symmetric.

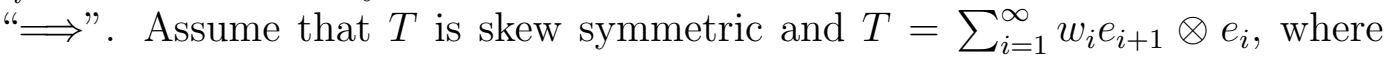
$\left\{e_{i}\right\}_{i=1}^{\infty}$ is an ONB of $\mathcal{H}$.

First we claim that card $\left\{i \in \mathbb{N}: w_{i}=0\right\}=\infty$. In fact, if not, then it is obvious that $T$ can be written as $T=A \oplus B$, where $A$ is acting on a finite dimensional Hilbert space and $B$ is a unilateral weighted shift with nonzero weights. Then $\operatorname{dim} \operatorname{ker} B=0, \operatorname{dim} \operatorname{ker} B^{*}=1$ and

$$
\begin{aligned}
\operatorname{dim} \operatorname{ker} T & =\operatorname{dim} \operatorname{ker} A+\operatorname{dim} \operatorname{ker} B=\operatorname{dim} \operatorname{ker} A=\operatorname{dim} \operatorname{ker} A^{*} \\
& <\operatorname{dim} \operatorname{ker} A^{*}+\operatorname{dim} \operatorname{ker} B^{*}=\operatorname{dim} \operatorname{ker} T^{*}<\infty .
\end{aligned}
$$

Since $T$ is skew symmetric, there exists a conjugation $C$ on $\mathcal{H}$ such that $C T C=$ $-T^{*}$. Then $\operatorname{dim} \operatorname{ker} T^{*}=\operatorname{dim} \operatorname{ker} T$, a contradiction. This proves the claim.

By the above claim, $T$ can be written as $T=\oplus_{i=1}^{\infty} A_{i}$, where each $A_{i}$ acting on a finite dimensional Hilbert space admits the following matrix representation

$$
A_{i}=\left[\begin{array}{ccccc}
0 & & & & \\
\mu_{1} & 0 & & & \\
& \ddots & \ddots & & \\
& & \mu_{k-2} & 0 & \\
& & & \mu_{k-1} & 0
\end{array}\right]
$$

relative to some ONB $\left\{e_{j}\right\}_{j=1}^{k}$ for the underlying space of $A_{i}$, where $\mu_{j} \neq 0$ for $1 \leq j \leq k-1$. So each $A_{i}$ is irreducible. By Corollary 2.8, if $\mathcal{M}$ is a nonzero minimal reducing subspace of $T$, then $\left.T\right|_{\mathcal{M}} \cong A_{i}$ for some $i \geq 1$. Moreover, it follows from [7, Thm. 3.1] that there exists no nonzero reducing subspace $\mathcal{N}$ of $T$ such that $\left.T\right|_{\mathcal{N}}$ is completely reducible.

Since $T$ is skew symmetric, it follows from Theorem 1.7 that $T=\oplus_{s \in \Lambda} B_{s}$, where each $B_{s}$ is either an irreducible SSO or $B_{s}=R \oplus\left(-R^{t}\right)$ with $R$ being irreducible and not skew symmetric. We shall show that each $B_{s}$ admits the desired form. 
Now we fix an $s \in \Lambda$.

Case 1. $B_{s}$ is an irreducible SSO. Then, by the discussion above, there exists $i$ such that $B_{s} \cong A_{i}$. Hence $A_{i}$ is skew symmetric. Note that $A_{i}$ admits the matrix representation (2.3). By Lemma 2.11, $A_{i}$ is a truncated weighted shift with symmetric weights and even rank. Then so is $B_{s}$.

Case 2. $B_{s}=R \oplus\left(-R^{t}\right)$ with $R$ being irreducible and not skew symmetric. Since $R$ is irreducible, it follows from Corollary 2.8 that $R \cong A_{i}$ for some $i$. Assume that $A_{i}$ admits the matrix representation (2.3). Then $A_{i}=\sum_{j=1}^{k-1} \mu_{j} e_{j+1} \otimes$ $e_{j}$. Define a conjugation $D$ on $\vee\left\{e_{j}: 1 \leq j \leq k\right\}$ by $D\left(\sum_{j=1}^{k} \alpha_{j} e_{j}\right)=\sum_{j=1}^{k} \overline{\alpha_{j}} e_{j}$. Note that

$$
\begin{aligned}
-D A_{i}^{*} D & =-D\left(\sum_{j=1}^{k-1} \overline{\mu_{j}} e_{j} \otimes e_{j+1}\right) D \\
& =-\sum_{j=1}^{k-1} \mu_{j} D\left(e_{j} \otimes e_{j+1}\right) D \\
& =-\sum_{j=1}^{k-1} \mu_{j} e_{j} \otimes e_{j+1}=\sum_{j=1}^{k-1}\left(-\mu_{j}\right) e_{j} \otimes e_{j+1} .
\end{aligned}
$$

Then $A_{i} \oplus\left(-D A_{i}^{*} D\right)$ can be written as

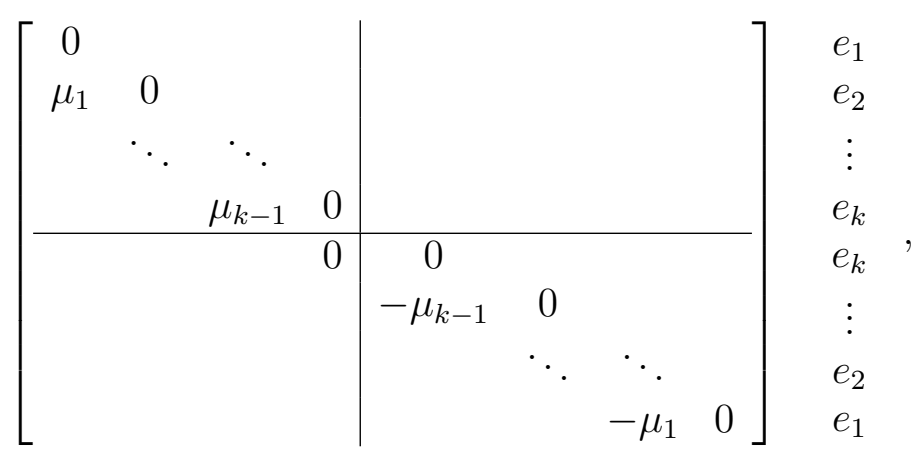

whence $A_{i} \oplus\left(-D A_{i}^{*} D\right)$ is a truncated weighted shift with symmetric weights and even rank. Noting that $B_{s}=R \oplus\left(-R^{t}\right) \cong A_{i} \oplus\left(-D A_{i}^{*} D\right)$, this completes the proof.

From the proof of Theorem 1.9, one can see the following result.

Corollary 2.13. Let $T$ be a direct sum of some finite-dimensional truncated weighted shifts. Then $T$ is skew symmetric if and only if $T$ can be written as $T=\oplus_{i \in \Lambda} T_{i}$, where each $T_{i}$ is a finite-dimensional truncated weighted shift with symmetric weights and even rank.

\section{Proof of Theorem 1.10}

Lemma 3.1. Let $T \in \mathcal{B}(\mathcal{H})$ and $T=U|T|$ be the polar decomposition of $T$. If $C$ is a conjugation on $\mathcal{H}$ and $C T C=-T^{*}$, then $C U|T|=|T| C U$. 
Proof. It is obvious that

$$
(C|T| C)^{2}=C|T|^{2} C=C T^{*} T C=T T^{*}=\left|T^{*}\right|^{2}=\left(U|T| U^{*}\right)^{2} .
$$

Then $C|T| C=U|T| U^{*}$ and hence $|T| C U=C U|T| U^{*} U$. Fix an $x \in \mathcal{H}$. If $x \in$ $(\operatorname{ker} U)^{\perp}$, then $|T| C U x=C U|T| U^{*} U x=C U|T| x$; if $x \in \operatorname{ker} U$, then $|T| C U x=$ $0=C U|T| x$. It follows that $|T| C U=C U|T|$.

Lemma 3.2. Let $T \in \mathcal{B}(\mathcal{H})$ with $T e_{i}=\alpha_{i} e_{i+1}$ for $i \in \mathbb{Z}$, where $\left\{e_{i}\right\}_{i \in \mathbb{Z}}$ is an ONB of $\mathcal{H}$ and $\alpha_{i}>0$ for all $i \in \mathbb{Z}$. Assume that $C$ is a conjugation on $\mathcal{H}$ and $C T C=-T^{*}$. Then

(i) $C e_{k} \in \vee\left\{e_{j}: \alpha_{j}=\alpha_{k-1}\right\}$ for all $k \in \mathbb{Z}$;

(ii) if $k, n \in \mathbb{Z}$ and $\left\langle C e_{k}, e_{n}\right\rangle \neq 0$, then $\left\langle C e_{k-j}, e_{n+j}\right\rangle \neq 0$ and $\alpha_{k-1-j}=\alpha_{n+j}$ for all $j \in \mathbb{Z}$.

Proof. (i) Assume that $T=U|T|$ is the polar decomposition of $T$. It is easy to check that $U e_{j}=e_{j+1}$ and $|T| e_{j}=\alpha_{j} e_{j}$ for all $j \in \mathbb{Z}$. Since $T$ is skew symmetric, it follows from Lemma 3.1 that $C U|T|=|T| C U$. Then, given $k \in \mathbb{Z}$, we have

$$
\begin{aligned}
\alpha_{k-1} C e_{k} & =\alpha_{k-1} C U e_{k-1}=C U \alpha_{k-1} e_{k-1} \\
& =C U|T| e_{k-1}=|T| C U e_{k-1}=|T| C e_{k},
\end{aligned}
$$

that is, $C e_{k} \in \operatorname{ker}\left(|T|-\alpha_{k-1}\right)=\vee\left\{e_{j}: \alpha_{j}=\alpha_{k-1}\right\}$.

(ii) Fix $i, j \in \mathbb{Z}$. Note that

$$
\begin{aligned}
\left\langle C e_{i-1}, e_{j+1}\right\rangle & =\frac{1}{\alpha_{i-1}}\left\langle C T^{*} e_{i}, e_{j+1}\right\rangle=\frac{-1}{\alpha_{i-1}}\left\langle T C e_{i}, e_{j+1}\right\rangle \\
& =\frac{-1}{\alpha_{i-1}}\left\langle C e_{i}, T^{*} e_{j+1}\right\rangle=\frac{-\alpha_{j}}{\alpha_{i-1}}\left\langle C e_{i}, e_{j}\right\rangle .
\end{aligned}
$$

It follows that $\left\langle C e_{i-1}, e_{j+1}\right\rangle=0$ if and only if $\left\langle C e_{i}, e_{j}\right\rangle=0$. Since $\left(C e_{k}, e_{n}\right) \neq 0$, one can deduce that $\left(C e_{k-l}, e_{n+l}\right) \neq 0$ for all $l \in \mathbb{Z}$. In view of (i), it follows that $\alpha_{k-1-l}=\alpha_{n+l}$ for $l \in \mathbb{Z}$.

Theorem 3.3. Let $T \in \mathcal{B}(\mathcal{H})$ with $T e_{i}=\alpha_{i} e_{i+1}$ for $i \in \mathbb{Z}$, where $\left\{e_{i}\right\}_{i \in \mathbb{Z}}$ is an ONB of $\mathcal{H}$ and $\alpha_{i} \neq 0$ for all $i \in \mathbb{Z}$. Then $T$ is skew symmetric if and only if there exists $k \in \mathbb{Z}$ such that $\left|\alpha_{2 k-j}\right|=\left|\alpha_{j-1}\right|$ for all $j \in \mathbb{Z}$.

Proof. Since $T$ is unitarily equivalent to the operator $\sum_{i \in \mathbb{Z}}\left|\alpha_{i}\right| e_{i+1} \otimes e_{i}$, we may directly assume that $\alpha_{i}>0$ for all $i \in \mathbb{Z}$.

"£". For $x \in \mathcal{H}$ with $x=\sum_{i \in \mathbb{Z}} \beta_{i} e_{i}$, we define

$$
C\left(\sum_{i \in \mathbb{Z}} \beta_{i} e_{i}\right)=\sum_{i \in \mathbb{Z}} \overline{\beta_{i}}(-1)^{i-k} e_{2 k-i} .
$$

One can check that $C$ is conjugate-linear, isometric and $C^{2} x=x$ for all $x \in \mathcal{H}$. Hence $C$ is a conjugation on $\mathcal{H}$ and, for $j \in \mathbb{Z}$, we have

$$
\begin{aligned}
C T C e_{j} & =(-1)^{j-k} C T e_{2 k-j}=(-1)^{j-k} \alpha_{2 k-j} C e_{2 k+1-j} \\
& =(-1)^{j-k} \alpha_{2 k-j}(-1)^{k+1-j} e_{j-1}=-\alpha_{2 k-j} e_{j-1} \\
& =-\alpha_{j-1} e_{j-1}=-T^{*} e_{j},
\end{aligned}
$$


that is, $C T C e_{j}=-T^{*} e_{j}$. Thus $C T C=-T^{*}$ and $T$ is skew symmetric.

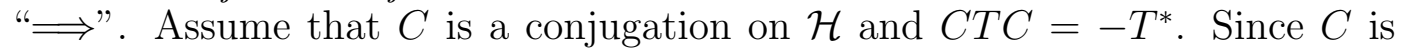
invertible, $C e_{0} \neq 0$ and there exists some $n \in \mathbb{Z}$ such that $\left\langle C e_{0}, e_{n}\right\rangle \neq 0$.

Claim. $n$ is even.

For a proof by contradiction, we assume that $n$ is odd. Then, by Lemma 3.2, $\left\langle C e_{\frac{n-1}{2}}, e_{\frac{n+1}{2}}\right\rangle \neq 0$. For convenience, we write $i=\frac{n-1}{2}$.

Assume that $C e_{i}=\sum_{j \in \mathbb{Z}} \lambda_{j} e_{j}$. Then, by the hypothesis, we have

$$
\lambda_{i+1} \neq 0 \quad \text { and } \quad\left\langle C e_{i}, e_{i+1}\right\rangle=\lambda_{i+1} .
$$

Moreover, we have

$$
\begin{aligned}
C e_{i+1} & =\frac{1}{\alpha_{i}} C T e_{i}=-\frac{1}{\alpha_{i}} T^{*} C e_{i} \\
& =-\frac{1}{\alpha_{i}} \sum_{j \in \mathbb{Z}} \lambda_{j} T^{*} e_{j}=-\frac{1}{\alpha_{i}} \sum_{j \in \mathbb{Z}} \lambda_{j} \alpha_{j-1} e_{j-1} .
\end{aligned}
$$

It follows that $\left\langle C e_{i+1}, e_{i}\right\rangle=-\frac{1}{\alpha_{i}} \cdot \lambda_{i+1} \cdot \alpha_{i}=-\lambda_{i+1}$. Noting that $C$ is a conjugation, it follows that $\left\langle C e_{i}, e_{i+1}\right\rangle=\left\langle C e_{i+1}, e_{i}\right\rangle=-\lambda_{i+1}$, contradicting (3.1). This proves the claim.

Since $\left\langle C e_{0}, e_{n}\right\rangle \neq 0$, it follows from Lemma 3.2 that $\alpha_{j-1}=\alpha_{n-j}$ for all $j \in \mathbb{Z}$. Set $k=\frac{n}{2}$. The desired result follows readily.

Theorem 3.4. Let $T$ be a bilateral weighted shift with weighted sequence $\left\{w_{i}\right\}_{i \in \mathbb{Z}}$. If $0<\operatorname{card}\left\{i \in \mathbb{Z}: w_{i}=0\right\}<\infty$, then $T$ is skew symmetric if and only if $T \cong A \oplus A^{*} \oplus B$, where $A$ is an injective unilateral weighted shift and $B$ is absent or $B$ is a finite direct sum of finite-dimensional truncated weighted shifts with symmetric weights and even rank.

Proof. "£". By Corollary 2.2, $A \oplus A^{*}$ is skew symmetric. From the proof for the sufficiency of Theorem 1.9, one can see that each finite-dimensional truncated weighted shifts with symmetric weights and even rank is skew symmetric. This proves the sufficiency.

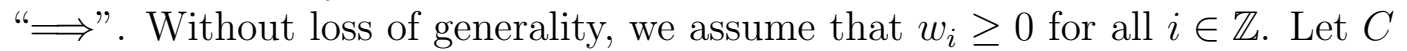
be a conjugation on $\mathcal{H}$ satisfying $C T C=-T^{*}$.

Case 1. card $\left\{i \in \mathbb{N}: w_{i}=0\right\}=1$. In this case, we may also assume that $w_{0}=0$. Then $\operatorname{ker} T^{m}=\vee\left\{e_{i}: 1-m \leq i \leq 0\right\}$ and $\operatorname{ker}\left(T^{*}\right)^{m}=\vee\left\{e_{i}: 1 \leq i \leq m\right\}$ for all $m \in \mathbb{N}$. Note that $C T^{m} C=\left(-T^{*}\right)^{m}$ and $C\left[\operatorname{ker}\left(T^{*}\right)^{m}\right]=\operatorname{ker} T^{m}$ for all $m \in \mathbb{N}$. Since $C$ is a conjugation, it follows that $C\left(\vee\left\{e_{m}\right\}\right)=\vee\left\{e_{1-m}\right\}$ for each $m \in \mathbb{N}$. On the other hand, $C$ preserves the norms of vectors, then there exists $\lambda_{m} \in \mathbb{C}$ with $\left|\lambda_{m}\right|=1$ such that $C e_{m}=\lambda_{m} e_{1-m}$ for $m \in \mathbb{N}$. Hence

$$
\begin{aligned}
w_{m-1} & =\left\|w_{m-1} C e_{m-1}\right\|=\left\|C T^{*} e_{m}\right\| \\
& =\left\|T C e_{m}\right\|=\left\|\lambda_{m} T e_{1-m}\right\|=\left\|w_{1-m} e_{2-m}\right\|=w_{1-m}
\end{aligned}
$$

for all $m \in \mathbb{N}$. That is, $w_{i}=w_{-i}$ for all $i \in \mathbb{N}$. Choose another onB $\left\{f_{i}\right\}_{i=1}^{\infty}$ of $\mathcal{H}$ and define $A \in \mathcal{B}(\mathcal{H})$ as $A f_{i}=w_{i} f_{i+1}$ for $i \geq 1$. Then it is obvious that $T \cong A \oplus A^{*}$. 
Case 2. $1<\operatorname{card}\left\{i \in \mathbb{N}: w_{i}=0\right\}<\infty$. In this case, there exist $m, n \in \mathbb{Z}$, $m<n$, such that $w_{n}=0=w_{m}$ and $w_{i} \neq 0$ for all $i>n$ or $i<m$. Denote $\mathcal{H}_{1}=\vee\left\{e_{i}: i \leq m\right\}, \mathcal{H}_{2}=\vee\left\{e_{i}: m<i \leq n\right\}$ and $\mathcal{H}_{3}=\vee\left\{e_{i}: i>n\right\}$. Then each $\mathcal{H}_{i}$ is a reducing subspace of $T$. For $1 \leq i \leq 3$, denote $T_{i}=\left.T\right|_{\mathcal{H}_{i}}$. Then $T_{1}^{*}, T_{3}$ are two injective unilateral weighted shifts and $T_{2}$ is a direct sum of some finitedimensional truncated weighted shifts. Note that $T_{2}$ is nilpotent and $T_{2}^{k}=0$, where $k=n-m$.

Claim. $T_{2}$ and $T_{1} \oplus T_{3}$ are both skew symmetric.

Obviously, it suffices to prove that $C\left(\mathcal{H}_{2}\right)=\mathcal{H}_{2}$. Arbitrarily choose an $x \in \mathcal{H}_{2}$. Since $\mathcal{H}_{2}=\operatorname{ker} T_{2}^{k} \subset \operatorname{ker} T^{k}$, we have $T^{k} x=0$. Noting that $C T^{k}=(-1)^{k}\left(T^{k}\right)^{*} C$, we have

$$
C x \in \operatorname{ker}\left(T^{k}\right)^{*}=\operatorname{ker}\left(T_{1}^{k}\right)^{*} \oplus \operatorname{ker}\left(T_{2}^{k}\right)^{*} \oplus \operatorname{ker}\left(T_{3}^{k}\right)^{*} .
$$

Since $\left(T_{1}^{k}\right)^{*}$ is injective and $T_{2}^{k}=0$, we obtain $C x \in \mathcal{H}_{2} \oplus \operatorname{ker}\left(T_{3}^{k}\right)^{*}$. On the other hand, we note that

$$
(-1)^{k} T^{k} C x=C\left(T^{k}\right)^{*} x=C\left(T_{2}^{k}\right)^{*} x=0 .
$$

Then $C x \in \operatorname{ker} T^{k}=\operatorname{ker} T_{1}^{k} \oplus \mathcal{H}_{2}$ and hence $C x \in \mathcal{H}_{2}$. Thus we have proved that $C\left(\mathcal{H}_{2}\right) \subset \mathcal{H}_{2}$, and it follows from $C^{2}=I$ that $C\left(\mathcal{H}_{2}\right)=\mathcal{H}_{2}$. This proves the claim.

By Corollary 2.13 and the proof in Case 1, it follows from the above claim that $T$ has the form as stated in the theorem.

Theorem 3.5. Let $T \in \mathcal{B}(\mathcal{H})$ be a weighted shift with weight sequence $\left\{w_{i}\right\}_{i \in \mathbb{Z}}$. If card $\left\{i \in \mathbb{Z}: w_{i}=0\right\}=\infty$, then $T$ is skew symmetric if and only if $T$ is an infinite direct sum of finite-dimensional truncated weighted shifts with symmetric weights and even rank.

Proof. From the proof for the sufficiency of Theorem 1.9, each finite-dimensional truncated weighted shifts with symmetric weights and even rank is skew symmetric. Thus the sufficiency is obvious. We need only prove the necessity.

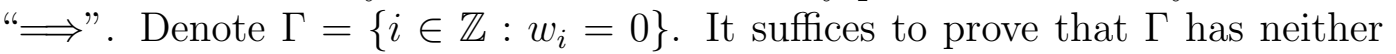
upper nor lower bound. In fact, if this holds, then, by rearranging the vectors in the ONB $\left\{e_{i}\right\}_{i \in \mathbb{Z}}$, one can see that $T$ is also a unilateral weighted shift. Then, by Theorem 1.9, one can obtain the conclusion.

For a proof by contradiction, we may directly assume that $\sup \Gamma<+\infty$ and $n=\sup \Gamma$ (the proof for the case "inf $\Gamma>-\infty$ " is similar). In this case, $T$ can be written as

$$
T=\left(\oplus_{i=1}^{\infty} A_{i}\right) \oplus B
$$

where each $A_{i}$ is an irreducible, nilpotent operator on some finite-dimensional space and $B$ is an injective unilateral weighted shift. Since $\operatorname{dim} \operatorname{ker} B=0$ and $\operatorname{dim} \operatorname{ker} B^{*}=1, B$ is not skew symmetric.

Assume that $C$ is a conjugation on $\mathcal{H}$ such that $C T C=-T^{*}$. Denote by $\mathcal{H}_{i}$ and $\mathcal{K}$ the underlying space of $A_{i}$ and $B$ respectively for $i \in \mathbb{N}$. Arbitrarily choose an $i \in \mathbb{N}$ and an $x \in \mathcal{H}_{i}$. Assume that $A_{i}^{k_{i}}=0$. Then $0=C\left(A_{i}^{k_{i}}\right)^{*} x=$ $C\left(T^{k_{i}}\right)^{*} x=(-1)^{k_{i}} T^{k_{i}} C x$ and hence $C x \in \operatorname{ker} T^{k_{i}} \subset \oplus_{j=1}^{\infty} \mathcal{H}_{j}$. Since $i \in \mathbb{N}$ and $x \in \mathcal{H}_{i}$ were arbitrarily chosen, we have $C\left(\oplus_{j=1}^{\infty} \mathcal{H}_{j}\right) \subset\left(\oplus_{j=1}^{\infty} \mathcal{H}_{j}\right)$. Noting that $C$ 
is a conjugation and $C^{2}=I$, we obtain $C\left(\oplus_{j=1}^{\infty} \mathcal{H}_{j}\right)=\oplus_{j=1}^{\infty} \mathcal{H}_{j}$ and $C(\mathcal{K})=\mathcal{K}$. Set $C_{1}=\left.C\right|_{\mathcal{K}}$. Then it is easy to verify that $C_{1}$ is a conjugation on $\mathcal{K}$ and it follows from $C T C=-T^{*}$ that $C_{1} B C_{1}=-B^{*}$. That is, $B$ is skew symmetric, a contradiction.

Remark 3.6. Summarizing the results of Theorems 3.3, 3.4 and 3.5, one can see Theorem 1.10.

\section{EXAMPLES}

In this section, we shall give several examples of completely reducible SSOs and irreducible SSOs. We remark that these examples are partially inspired by several examples of special CSOs given in [18].

Example 4.1. We shall construct a completely reducible operator $T$ which is skew symmetric and not normal. Let $\mathcal{H}=L^{2}([-1,1], \mathrm{d} m)$ and $A$ be the "multiplication by $t$ " operator on $\mathcal{H}$, where $\mathrm{d} m$ denotes the Lebesgue measure. Then $A$ is self-adjoint and completely reducible. By [20, Thm. 1.11], $A$ is skew symmetric. For the reader's convenience, we explain this in detail. For $f \in \mathcal{H}$, define $(C f)(t)=\overline{f(-t)}$. Then one can verify that $C$ is a conjugation on $\mathcal{H}$. So

$$
(C A f)(t)=C(t f)(t)=-t \overline{f(-t)}=-(A C(f))(t), \quad \forall f \in \mathcal{H},
$$

that is, $C A=-A C$. This shows that $A$ is skew symmetric. Define a conjugation $D$ on $\mathcal{H}^{(2)}$ as

Set

$$
D=\left[\begin{array}{cc}
0 & C \\
C & 0
\end{array}\right] \mathcal{H}
$$

$$
T=\left[\begin{array}{cc}
0 & A \\
0 & 0
\end{array}\right] \stackrel{\mathcal{H}}{\mathcal{H}}
$$

It is obvious that $T$ is not normal. Now compute to see

$$
D T=\left[\begin{array}{cc}
0 & 0 \\
0 & C A
\end{array}\right]=\left[\begin{array}{cc}
0 & 0 \\
0 & -A C
\end{array}\right]=-\left[\begin{array}{cc}
0 & 0 \\
A & 0
\end{array}\right]\left[\begin{array}{cc}
0 & C \\
C & 0
\end{array}\right]=-T^{*} D
$$

Thus we have proved that $T$ is skew symmetric.

Now we shall prove that $T$ is completely reducible. For convenience, we write

$$
T=\left[\begin{array}{cc}
0 & A \\
0 & 0
\end{array}\right] \mathcal{H}_{1}
$$

where $\mathcal{H}_{1}=\mathcal{H}_{2}=\mathcal{H}$. Let $P \in \mathcal{B}\left(\mathcal{H}^{(2)}\right)$ be an orthogonal projection commuting with $T$. Assume that

$$
P=\left[\begin{array}{ll}
P_{1,1} & P_{1,2} \\
P_{2,1} & P_{2,2}
\end{array}\right] \mathcal{H}_{1}
$$

Since $\operatorname{ker} T=\mathcal{H}_{1}$, ker $T^{*}=\mathcal{H}_{2}$ are hyperinvariant subspace of $T$, we obtain $P\left(\mathcal{H}_{1}\right) \subset \mathcal{H}_{1}$ and $P\left(\mathcal{H}_{2}\right) \subset \mathcal{H}_{2}$. It follows that $P_{1,2}=P_{2,1}=0$.

Note that

$$
|T|=\left[\begin{array}{cc}
0 & 0 \\
0 & |A|
\end{array}\right] \mathcal{H}_{1} \mathcal{H}_{2}, \quad\left|T^{*}\right|=\left[\begin{array}{cc}
|A| & 0 \\
0 & 0
\end{array}\right] \mathcal{H}_{1}
$$


Since $P|T|=|T| P$ and $P\left|T^{*}\right|=\left|T^{*}\right| P$, it follows from (4.1) that $P_{i, i}|A|=|A| P_{i, i}$, $i=1,2$. Noting that $A$ is self-adjoint, we obtain $P_{i, i} A^{2}=A^{2} P_{i, i}, i=1,2$. On the other hand, since $P T=T P$, we obtain $P_{1,1} A=A P_{2,2}$. Hence $P_{1,1} A^{2}=A^{2} P_{2,2}$. Hence $A^{2} P_{1,1}=A^{2} P_{2,2}$. Furthermore we obtain $P_{1,1}=P_{2,2}$. Thus we have proved that each orthogonal projection $P$ commuting with $T$ has the form $Q^{(2)}$, where $Q$ is an orthogonal projection on $\mathcal{H}$ commuting with $A$. Since $A$ is completely reducible, we deduce that $T$ is completely reducible.

Example 4.2. We shall construct an irreducible SSO on an infinite dimensional Hilbert space. Let $S \in \mathcal{B}(\mathcal{H})$ be the unilateral shift defined by $S e_{i}=e_{i+1}$ for $i \geq 1$, where $\left\{e_{i}\right\}_{i=1}^{\infty}$ is an ONB of $\mathcal{H}$. Define $F \in \mathcal{B}(\mathcal{H})$ as

$$
F e_{1}=e_{2}, \quad F e_{2}=e_{1}, \quad F e_{i}=0, \quad \forall i \geq 3 .
$$

Set

$$
T=\left[\begin{array}{cc}
S^{*} & F \\
0 & S
\end{array}\right] \mathcal{H}_{1}
$$

where $\mathcal{H}_{1}=\mathcal{H}_{2}=\mathcal{H}$. Then it is easy to verify that $T$ is irreducible. Now it remains to check that $T$ is skew symmetric.

For $x \in \mathcal{H}$ with $x=\sum_{i} \alpha_{i} e_{i}$, define $C x=\sum_{i} \overline{\alpha_{i}}(-1)^{i} e_{i}$. Then one can check that $C$ is a conjugation on $\mathcal{H}$. For each $i \geq 1$, one can see that

$$
C S C e_{i}=(-1)^{i} C S e_{i}=(-1)^{i} C e_{i+1}=(-1)^{2 i+1} e_{i+1}=-S e_{i},
$$

which implies $C S C=-S$. Thus we also have $C S^{*} C=-S^{*}$. On the other hand, one can check that

$$
\begin{gathered}
C F C e_{1}=-C F e_{1}=-C e_{2}=-e_{2}=-F^{*} e_{1}, \\
C F C e_{2}=C F e_{2}=C e_{1}=-e_{1}=-F^{*} e_{2}
\end{gathered}
$$

and

$$
C F C e_{i}=(-1)^{i} C F e_{i}=0=-F^{*} e_{i}, \quad \forall i \geq 3 .
$$

Thus we have $C F C=-F^{*}$.

Define a conjugation $D$ on $\mathcal{H}^{(2)}$ as

$$
D=\left[\begin{array}{cc}
0 & C \\
C & 0
\end{array}\right] \mathcal{H}_{1}
$$

A direct computation shows that

$$
D T=\left[\begin{array}{cc}
0 & C S \\
C S^{*} & C F
\end{array}\right]=\left[\begin{array}{cc}
0 & -S C \\
-S^{*} C & -F^{*} C
\end{array}\right]=-\left[\begin{array}{cc}
S & 0 \\
F^{*} & S^{*}
\end{array}\right]\left[\begin{array}{cc}
0 & C \\
C & 0
\end{array}\right]=-T^{*} D .
$$

Hence $T$ is an irreducible SSO.

Example 4.3. We shall give an SSO $T$ which is reducible but does not admit a nontrivial reducing subspace $\mathcal{M}$ of $T$ such that $\left.T\right|_{\mathcal{M}}$ is skew symmetric.

Let $S \in \mathcal{B}(\mathcal{H})$ be the unilateral shift defined by $S e_{i}=e_{i+1}$ for $i \geq 1$, where $\left\{e_{i}\right\}_{i=1}^{\infty}$ is an ONB of $\mathcal{H}$. By Lemma 2.1, the operator $T:=S^{*} \oplus S$ is skew symmetric. Arbitrarily choose a nontrivial reducing subspace $\mathcal{M}$ of $T$. We shall prove that $\left.T\right|_{\mathcal{M}}$ is not skew symmetric. Noting that $S, S^{*}$ are irreducible and $S$ is not unitarily equivalent to $S^{*}$, it follows from Proposition 2.4 that either 
$\mathcal{M}=\mathcal{H}_{1}$ or $\mathcal{M}=\mathcal{H}_{2}$. Thus we have either $\left.T\right|_{\mathcal{M}}=S^{*}$ or $\left.T\right|_{\mathcal{M}}=S$. So it remains to check that $S$ is not skew symmetric.

In fact, if not, then there is a conjugation $C$ on $\mathcal{H}$ such that $C S C=-S^{*}$. So

$$
\begin{aligned}
0 & =\operatorname{dim} \operatorname{ker}\left(S+\frac{1}{2}\right)=\operatorname{dim} \operatorname{ker} C\left(S+\frac{1}{2}\right) C \\
& =\operatorname{dim} \operatorname{ker}\left(-S^{*}+\frac{1}{2}\right)=\operatorname{dim} \operatorname{ker}\left(S^{*}-\frac{1}{2}\right)=1,
\end{aligned}
$$

which is absurd.

Acknowledgement. The author sincerely thanks the referee for his careful reading of the manuscript and invaluable comments which were very helpful in improving this paper. The author acknowledges partial support of National Natural Science Foundation of China (11101177, 11271150, 11326103).

\section{REFERENCES}

1. N.I. Akhiezer and I.M. Glazman, Theory of linear operators in Hilbert space, Dover Publications, Inc., New York, 1993.

2. J.L. Boersema, The range of united K-theory, J. Funct. Anal. 235 (2006), no. 2, 701-718.

3. J.A. Cima, W.T. Ross, and W.R. Wogen, Truncated Toeplitz operators on finite dimensional spaces, Oper. Matrices 2 (2008), no. 3, 357-369.

4. A. Connes, A factor not anti-isomorphic to itself, Ann. Math. (2) 101 (1975), 536-554.

5. A. Connes, Sur la classification des facteurs de type II, C. R. Acad. Sci. Paris Sér. A-B 281 (1975), no. 1, Aii, A13-A15.

6. J.B. Conway, A course in operator theory, Graduate Studies in Mathematics, vol. 21, American Mathematical Society, Providence, RI, 2000.

7. J.S. Fang, C.L. Jiang, and P.Y. Wu, Direct sums of irreducible operators, Studia Math. 155 (2003), no. 1, 37-49.

8. N.S. Feldman, Essentially subnormal operators, Proc. Amer. Math. Soc. 127 (1999), no. 4, 1171-1181.

9. S.R. Garcia and D.E. Poore, On the norm closure of the complex symmetric operators: compact operators and weighted shifts, J. Funct. Anal. 264 (2013), no. 3, 691-712.

10. S.R. Garcia and M. Putinar, Complex symmetric operators and applications, Trans. Amer. Math. Soc. 358 (2006), no. 3, 1285-1315.

11. S.R. Garcia and M. Putinar, Complex symmetric operators and applications. II, Trans. Amer. Math. Soc. 359 (2007), no. 8, 3913-3931.

12. S.R. Garcia and W.R. Wogen, Complex symmetric partial isometries, J. Funct. Anal. 257 (2009), no. 4, 1251-1260.

13. S.R. Garcia and W. Ross, Recent progress on truncated Toeplitz operators, Fields Inst. Commun. 65 (2013), 275-319.

14. S.R. Garcia, W. Ross, and W.R. Wogen, $C^{*}$-algebras generated by truncated Toeplitz operators, Oper. Theory. Adv. Appl. 236 (2013), 181-192.

15. I.M. Glazman, An analogue of the extension theory of Hermitian operators and a nonsymmetric one-dimensional boundary problem on a half-axis, Dokl. Akad. Nauk SSSR (N.S.) 115 (1957), 214-216 (Russian).

16. I.M. Glazman, Direct methods of qualitive spectral analysis of singular differential operators, Daniel Davey, 1965.

17. K. Guo, Y. Ji, and S. Zhu, A $C^{*}$-algebra approach to complex symmetric operators, Trans. Amer. Math. Soc. (to appear)

18. K. Guo and S. Zhu, A canonical decomposition of complex symmetric operators, J. Operator Theory (to appear) 
19. C.G. Li and T.T. Zhou, Skew symmetry of a class of operators, Banach J. Math. Anal. 8 (2014), no. 1, 279-294.

20. C.G. Li and S. Zhu, Skew symmetric normal operators, Proc. Amer. Math. Soc. 141 (2013), no. 8, 2755-2762.

21. N.C. Phillips and M.G. Viola, A simple separable exact $C^{*}$-algebra not anti-isomorphic to itself, Math. Ann. 355 (2013), no. 2, 783-799.

22. D. Sarason, Algebraic properties of truncated Toeplitz operators, Oper. Matrices 1 (2007), no. 4, 491-526.

23. N.A. Sedlock, Properties of truncated Toeplitz operators, ProQuest LLC, Ann Arbor, MI, 2010, Thesis (Ph.D.)-Washington University in St. Louis.

24. N.A. Sedlock, Algebras of truncated Toeplitz operators, Oper. Matrices 5 (2011), no. 2, 309-326.

25. P.J. Stacey, Real structure in unital separable simple $C^{*}$-algebras with tracial rank zero and with a unique tracial state, New York J. Math. 12 (2006), 269-273.

26. P.J. Stacey, Antisymmetries of the CAR algebra, Trans. Amer. Math. Soc. 363 (2011), no. 12, 6439-6452, With an appendix by J. L. Boersema and N. C. Phillips.

27. S.M. Zagorodnyuk, On a J-polar decomposition of a bounded operator and matrices of Jsymmetric and J-skew-symmetric operators, Banach J. Math. Anal. 4 (2010), no. 2, 11-36.

28. S.M. Zagorodnyuk, On the complex symmetric and skew-symmetric operators with a simple spectrum, SIGMA Symmetry Integrability Geom. Methods Appl. 7 (2011), 1-9.

29. S. Zhu and C.G. Li, Complex symmetric weighted shifts, Trans. Amer. Math. Soc. 365 (2013), no. 1, 511-530.

30. S. Zhu, Approximate unitary equivalence to skew symmetric operators, Complex Anal. Oper. Theory, 2014, doi: 10.1007/s11785-014-0369-z

Department of Mathematics, Jilin University, Changchun 130012, P. R. China. E-mail address: zhusen@jlu.edu.cn 